\title{
The Specific and Nonspecific Effects of Tai Chi and Its Possible Central Responses: A Protocol of Neuroimaging Study
}

\author{
Tianyu Liu $\mathbb{D}^{1,2}$ Yuke Teng $\mathbb{D}^{2,3}$ Sha Yang $\mathbb{D}^{2,3}$ Yuyi Guo, ${ }^{2,3}$ Tao Yin, ${ }^{2,3}$ Jingwen Chen, ${ }^{2,3}$ \\ Rongtao Ying $\mathbb{D},{ }^{3}$ Zhaoxuan $H e \mathbb{D}^{2,3}$ Shuguang $Y u^{3}$ Jianwei $W u\left(\mathbb{D},{ }^{4}\right.$ and Fang Zeng $\mathbb{D}^{2,3}$ \\ ${ }^{1}$ School of Sport, Chengdu University of Traditional Chinese Medicine, Chengdu, Sichuan, China \\ ${ }^{2}$ Acupuncture and Brain Science Research Center, Chengdu University of Traditional Chinese Medicine, Chengdu, \\ Sichuan, China \\ ${ }^{3}$ School of Acupuncture and Tuina, The 3rd Teaching Hospital, Chengdu University of Traditional Chinese Medicine, Chengdu, \\ Sichuan, China \\ ${ }^{4}$ School of Chinese Classics, Chengdu University of Traditional Chinese Medicine, Chengdu, Sichuan, China
}

Correspondence should be addressed to Jianwei Wu; wujianwei@cdutcm.edu.cn and Fang Zeng; zeng_fang@126.com

Received 6 September 2020; Revised 12 January 2021; Accepted 18 January 2021; Published 19 February 2021

Academic Editor: Liye Zou

Copyright (c) 2021 Tianyu Liu et al. This is an open access article distributed under the Creative Commons Attribution License, which permits unrestricted use, distribution, and reproduction in any medium, provided the original work is properly cited.

Tai Chi has been proven to be a safe and effective assistant therapy for healthcare and disease treatment. However, whether the adjuvant therapeutic effect of Tai Chi is general or disease-oriented remains uncertain. This trial focuses on exploring the specific and nonspecific effects of Tai Chi and its potential central responses. The results will deepen our understanding of the characteristics of Tai Chi exercise for adjuvant therapeutic effects and promote its application in the clinic. In this neuroimaging trial, 40 functional constipation (FC) patients and 40 healthy subjects (HS) will be recruited and will receive 10 weeks of Tai Chi exercise. The motor function, respiratory function, stool-related symptoms, quality of life, and emotional state of the participants will be evaluated at the baseline, the 5-week Tai Chi practice, and the end of practice. The potential changes in the heart rate variability and the cerebral function will be recorded by the $24 \mathrm{~h}$ dynamic electrocardiogram at the baseline and the functional magnetic resonance imaging at the end of practice. The possible correlations among the clinical variables, the heart rate variability, and the cerebral activity alterations in FC patients and HS will be analyzed. The healthcare and therapeutic effects of Tai Chi exercise might consist of the specific and nonspecific effects. This study provides not only a new perspective for understanding Tai Chi but also a new approach for investigating the mind-body exercise. This trial was registered in the Chinese Clinical Trial Registry (http://www.chictr.org.cn/showproj.aspx?proj=33243) on 28 November 2018 (registration number: ChiCTR1800019781; protocol version number: V1.0). This trial is currently in the stage of recruiting patients. The first patient was included on 1 December 2018. To date, 18 FC patients and 20 HS have been included. Recruitment will be completed in December 2020.

\section{Introduction}

Tai Chi is a traditional mind-body exercise, which originated from ancient China and widely spread all over the world. It is reported that there are 5 and 150 million Tai Chi practitioners in China and the whole world, respectively. Tai Chi has dual effects on healthcare and treatment. For healthy subjects (HS), the healthcare effects of Tai Chi in strengthening muscle strength [1], improving physical flexibility [2], enhancing the ability to balance and control [2], increasing vital capacity [3], reducing stress [4], and others have long been identified. For patients, the therapeutic effects of Tai Chi for treating multiple chronic diseases including osteoarthritis [5], hypertension [6], type 2 diabetes mellitus [7], coronary heart disease [8], and chronic obstructive pulmonary disease [9] have also been proven by a number of studies. These studies indicated that Tai Chi was widely involved in the prevention, treatment, and rehabilitation of various diseases. Does Tai Chi have different effects on the same organ/system in different practitioners? For example, a systematic review and meta-analysis showed that mind-body interventions, including Tai Chi, were effective 
in alleviating gastrointestinal symptoms and improving the quality of life (QOL) of patients [10]. Does Tai Chi have different gastrointestinal modulation effects between patients with gastrointestinal disorders and HS? In other words, is the gastrointestinal regulation of Tai Chi diseaseoriented and influenced by individual physical condition? However, majority of the Tai Chi-related studies were performed either on patients or HS, and few studies were performed on both patients and HS to investigate the influence of physical conditions on the effect of Tai Chi.

On the basis of the characteristics of Tai Chi and previous researches, we predict that the effects of Tai Chi include two aspects: the relatively specific effects and nonspecific effects. The nonspecific effects mainly refer to the modulating effects on human motor function (muscle strength, physical flexibility, etc.) and respiratory function, especially vital capacity. The nonspecific effects might also manifest as maintaining the normal function of organs/ systems, keeping the body in a relatively balanced and coordinated state. While the relatively specific effects of Tai Chi are disease-oriented, meaning that when the body is in a pathological state, Tai Chi practice might reduce the hyperactive function to improve the hypoactive function.

To test the hypothesis, we design this neuroimaging study. In this study, both functional constipation (FC) patients and HS were selected as participants to investigate the specific effects (gastrointestinal function) and nonspecific effects (motor function and respiratory function) of Tai Chi. FC is a common functional gastrointestinal disorder (FGID) with high prevalence. It is characterized by various constipation-related symptoms, including reduced defecation, defecation stress, hard stools, and uncomfortable abdominal muscle in the absence of evident organic or structural reasons for these symptoms. A randomized control trial indicated that Tai Chi practice could significantly improve the constipation symptom of patients with chronic FC [11].

This study aims to (1) investigate the nonspecific effects of Tai Chi by comparing the influence of Tai Chi practice on motor function and respiratory function between FC patients and HS using the lower-extremity muscle strength test, functional balance tests, and vital capacity test; (2) investigate the specific effects of Tai Chi from three aspects, including gastrointestinal symptom, autonomic nervous activity, and emotional state; and (3) explore the potential central responses of Tai Chi's specific and nonspecific effects by functional magnetic resonance imaging (fMRI).

\section{Methods and Analysis}

2.1. Study Design. The trial is designed as a neuroimaging trial that focuses on specific and nonspecific effects of Tai Chi and its potential central responses. A total of $40 \mathrm{FC}$ patients and $40 \mathrm{HS}$ will be recruited. Fifteen participants in each group will be randomly selected to undergo MRI scan. The study procedure is outlined in Figure 1.

2.2. Sample Size Calculation. We will investigate the therapeutic effects of Tai Chi for FC patients. According to our preliminary study from which we recruited 10 FC patients with the Tai Chi intervention, the mean improvement of weekly complete spontaneous bowel movements (CSBMs) of Tai Chi was $1.67 \pm 0.89$ times. A systematic review [12] focusing on the management of FC reported the mean improvement of weekly CSBMs of polyethylene glycol (the firstline medication of FC) was 1.8 times. We hypothesize that the therapeutic effects of Tai Chi are not less than the polyethylene glycol. Considering $\alpha=0.05,1-\beta=0.8$, the study design required a sample size of 36 for each group with the onesample noninferiority test [13], with a drop-out rate of $10 \%$. A total of 40 patients with FC and $40 \mathrm{HS}$ will be finally recruited.

$$
N=\left(\sigma \frac{Z_{1-\alpha}+Z_{1-\beta}}{\mu-\mu o-\delta}\right)^{2} \text {. }
$$

2.3. Patients with FC. Patients who match the inclusion criteria will be recruited. FC patients will be diagnosed by two gastroenterologists in the digestion department of the Hospital of CDUTCM, according to the Rome IV Diagnostic Criteria for FC [14]. Each FC patient will undergo a careful physical examination including a routine blood test, routine urine test, routine stool test, blood biochemical test (ALT, AST, BUN, Scr), transabdominal ultrasound, and dynamic electrocardiogram. The inclusion criteria and exclusion criteria of FC patients were as same as our previous study [15].

2.3.1. Inclusion Criteria. Patients will be included in the study if they (1) reach the Rome IV Diagnostic Criteria for FC [14], (2) are right-handers and the age range is between 18 and 35, (3) have less than three CSBMs every week, (4) the Cleveland Constipation Score (CCS) score $>10$, and (5) provide a written informed consent form.

2.3.2. Exclusion Criteria. Patients will be excluded from the study if they (1) are pregnant women, or plan to be pregnant within 3 months, or are breast-feeding women, (2) are incapable of sports, (3) have a history of head trauma and loss of consciousness, (4) have diabetes or serious cardiovascular, neurological, psychiatric, renal, or respiratory disease, (5) have moderate or serious depression and anxiety, (6) cannot keep silent for 20 minutes while lying down, (7) have any contraindications to $\mathrm{fMRI}$ scanning including the presence of metal stent, metal denture, or claustrophobia, (8) have taken other exercises (including meditation and yoga) that may improve constipation over 30 minutes every week in the last 3 months, (9) have taken gastrointestinal motility medicine, nonsteroidal anti-inflammatory medicine, and steroids in the last 15 days, (10) have received other treatments (including surgery, diet modification, biofeedback, or probiotics) in the last month, or (11) have participated in any other clinical trials in the past 3 months.

2.4. Healthy Subjects. Those who match the inclusion criteria will be recruited and will receive the same physical examination with the FC patients. 


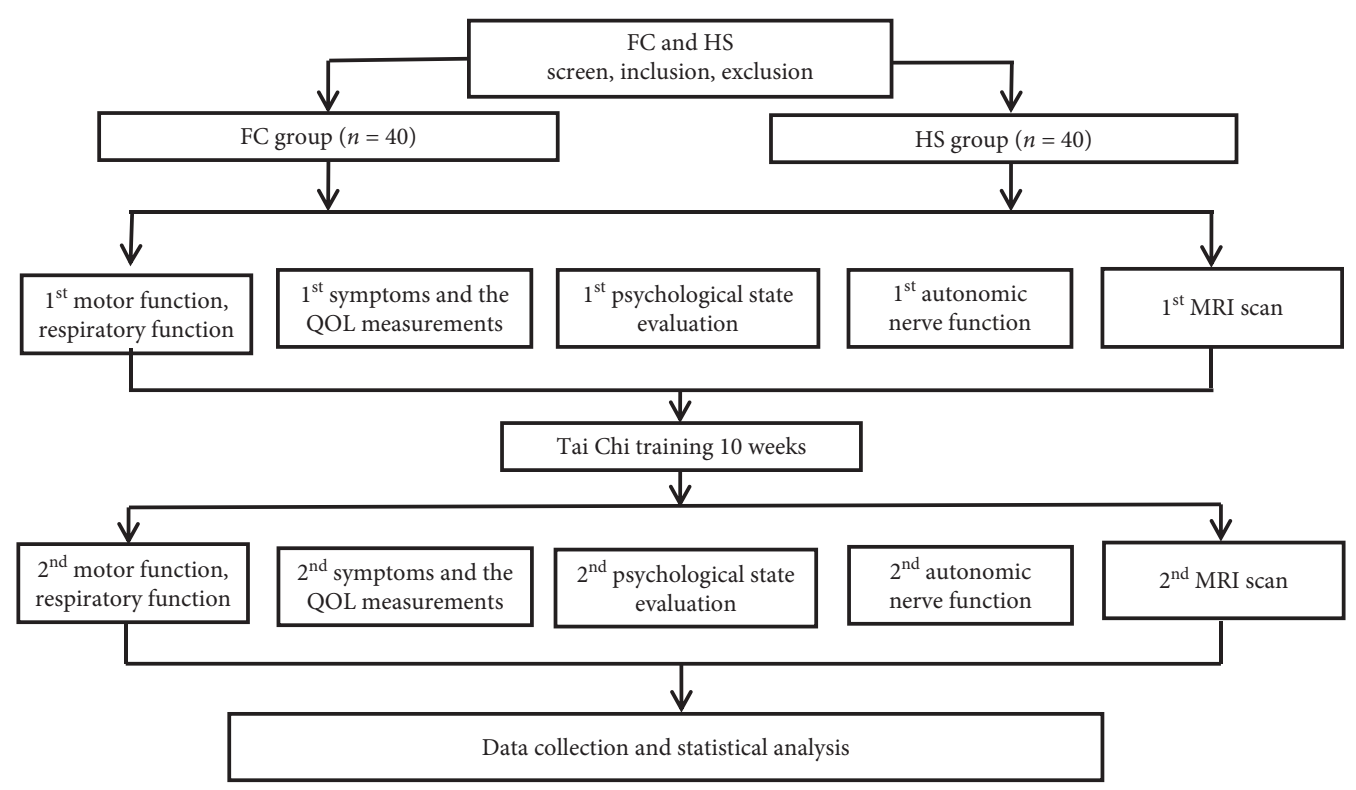

Figure 1: Study flowchart. This is a neuroimaging trial which focuses on specific and nonspecific effects of Tai Chi and its potential central responses. Totally, 40 eligible FC patients and $40 \mathrm{HS}$ will be recruited. Fifteen participants in each group will be randomly selected to undergo functional magnetic resonance imaging (fMRI) scan.

2.4.1. Inclusion Criteria. Subjects will be included if they (1) are right-handers and aged 18-35 years, (2) have no abnormalities during the physical examinations, (3) have passed the National Student Physical Health Standard test, and (4) provide written informed consent.

2.4.2. Exclusion Criteria. The exclusion criteria for HS are the same as the criteria for FC patients (see Section 2.3.2). Moreover, those who have possible organic diseases, psychological disorders, and gastrointestinal symptoms and signs are also excluded.

2.5. Recruitment Strategy. The participants will be recruited from the Chengdu University of Traditional Chinese Medicine (CDUTCM) by delivering leaflets inside the campus, posting advertisements in billboards, posting at Acupuncture and Brain Science Research Center and CDUTCM (http://cdutcm.edu.cn/) websites, and posting in our WeChat public account.

2.6. Intervention. The participants will undergo 40 sessions with 24-style Tai Chi. The exercise of Tai Chi will be performed four times a week (Monday, Tuesday, Thursday, and Friday) and will last for 10 weeks [15]. Each exercise session lasts for an hour, including 10-minute warm-up, 40-minute Tai Chi exercise, and 10-minute relaxation.

Before the formal intervention, participants were involved in motor learning and strength training under the guidance of two professional Tai Chi instructors. During the intervention, they exercise strictly under the 24-style Tai Chi exercise standard operating procedure (SOP), which was formulated by two Tai Chi experts. In each session of the exercise, the instant heart rate will be counted both at the pre- and posttrial, and the sports self-rating scale will also be filled out. Participants who did not come will be recorded. The training will be considered effective when the rating of perceived exertion (RPE) of the subjects reaches 4-6 levels in each session, and training times must not be less than $80 \%$ (32 times) of total training times.

During the treatment period, participants are usually not recommended to use concomitant care or interventions. However, if required (such as, aggravation of the patient's condition), participants will be permitted to use extra osmotic laxatives. The type and dosage of medication used should be recorded in the case report forms (CRFs).

2.7. Outcome Measurements. Measurements will be evaluated by independent assessors who have been trained prior to the study. All results will be recorded whether or not the participants completed the study. The outcome assessors will be independent of the research team and will not be told about group allocation, so as to ensure the object evaluations of the trial.

2.7.1. General Information Collection. Same as our previous study [15], general information including the demographic data and vital signs will be collected. The demographic data include age, gender, education level, nationality, and body mass index (BMI). The vital signs include body temperature, heart rate, respiratory rate, and blood pressure. Among them, the BMI will be calculated at the baseline and the end of the invention, whereas the vital signs of each patient will be immediately recorded after every training.

According to our hypothesis, the nonspecific effects of Tai Chi include motor function, vital capacity, and psychological state regulation and the specific effects of Tai Chi 
for FC patients reflects in relieving gastrointestinal symptoms and modulating autonomic nerve function.

\subsubsection{Nonspecific Effects: Motor Function, Vital Capacity,} and Psychological State. The lower-extremity muscle strength, functional balance tests, and the vital capacity test will be collected at the baseline, the median of intervention (after 5 weeks of exercise), and at the end of the intervention. The lower-extremity muscle strength will be measured using a hand-held isometric dynamometer (Micro FET3; Hoggan Health Industries, Draper, UT, United States). Functional balance tests include the Berg Balance Scale (BBS) [16], timed up-and-go (TUG) test, and functional reach test [17].

During the vital capacity test, the forced expiratory volume in one second (FEV1), forced vital capacity (FVC), and FEV1/FVC ratio will be measured using a Super Spiro spirometer (MicroMedical, Rochester, Kent, UK) in resting status. The peak expiratory flow rate (PEFR) will be assayed using a peak flow meter.

To assess the psychological state of all participants, we collected the following metrics at the baseline, the median of intervention, and the end of intervention: the self-rating depression scale (SDS) [18], the self-rating anxiety scale (SAS) [19], and the Eysenck Personality Questionnaire (EPQ) [20].

2.7.3. Specific Effects: Gastrointestinal Symptoms and Autonomic Nerve Function. To evaluate the changes of the gastrointestinal symptoms and QOL of participants, we performed the following measurements at the baseline, the median of intervention, and the end of intervention: CCS [21], patient assessment of constipation symptoms (PACSYM) [22], patient assessment of constipation QOL questionnaire (PAC-QOL) [23], RPE [24], and MOS 36-item short-form health survey (SF-36) [25]. Also, the evaluation of constipation diary, including CSBMs per week, the fecal character, and the difficulty degree of defecation, will be measured once a week for 10 times.

To evaluate the autonomic nervous function, we selected the heart rate variability (HRV). All participants will be assessed during the 24-h HRV at the baseline and the end of the intervention. The metrics of HRV include standard deviation of $\mathrm{NN}$ intervals (SDNN), standard deviation of sequential 5-min RR interval means (SDANN), and root mean square successive difference (RMSSD). The device used is a dynamic electrocardiogram (ct-086S; BENE WARE, Hangzhou, China).

2.8. MRI Data Acquisition. MRI data will be collected on 15 participants in each group at the baseline and the end of intervention. The acquisition parameter will be consistent with our previous article [15].

The MRI scan includes three sequences: a high-resolution 3-dimensional T1-weighted imaging (3D-T1W1), a blood oxygenation level-dependent functional MRI (BOLDfMRI), and a diffusion tensor imaging (DTI) sequence. Scanning will start in the morning after overnight fasting.
Subjects who are selected will undergo MRI scan with a $3.0 \mathrm{~T}$ magnetic resonance scanner (Siemens, Germany). The 3DT1WI scan parameters will be as follows: repetition time/ echo time: $1900 \mathrm{~ms} / 2.26 \mathrm{~ms}$; slice thickness: $1 \mathrm{~mm}$, slice number: 176 , matrix size: $128 \times 128$, and the view field: $256 \times 256 \mathrm{~mm}^{2}$. The BOLD-fMRI scanning parameters are as follows: repetition time/echo time: $2000 \mathrm{~ms} / 30 \mathrm{~ms}$; flip angle: $90^{\circ}$; slice number: 30 ; matrix size: $128 \times 128$; view field: $240 \times 240 \mathrm{~mm}^{2}$; slice thickness: $5 \mathrm{~mm}$; and total volume: 240 . DTI data are as follows: view field: $240 \times 240 \mathrm{~mm}^{2}$; repetition time/echo time: $6800 \mathrm{~ms} / 93 \mathrm{~ms}$; matrix size: $128 \times 128$; and slice thickness: $3 \mathrm{~mm}$, seamless. Two diffusion-weighted sequences will be acquired using gradient values b: $1000 \mathrm{~s} /$ $\mathrm{mm}^{2}$ and b: 0 , with diffusion-sensitizing gradients used in 64 noncollinear directions.

2.9. Data Management. The clinical data will be managed with printed and electronic CRFs. CRFs will be entered parallelly and will only be available to the outcome assessors. The Evidence-based Medicine Center of the CDUTCM will be responsible for monitoring the study and data every 3 months.

\subsection{Statistical Analysis}

2.10.1. Clinical Data Analysis. Clinical data will be analyzed by independent statisticians who do not know the test procedure based on the principles of intention to treat (ITT) and per protocol. The statistical significance threshold has $p$ value $<0.05$. The results of the participants who failed to complete the study will be considered no different from the baseline data in ITT analysis. All continuous variables will be presented as mean \pm standard deviation. The categorical variables will be described in percentage (\%). The clinical data in the two groups (FC group and HS group) will be compared with two-sample $t$-tests, and the comparisons of the baseline and after intervention in each group will be compared with a paired sample $t$-test. Nonparametric tests (Mann-Whitney $U$ test) will be used to compare nonnormally distributed clinical data, and the $\chi^{2}$ test or Fisher's test will be used to compare categorical variables.

2.10.2. Functional MRI Data Analysis. For fMRI scans, all preprocessing steps will be performed using DPABI software based on MATLAB. The main analytical methods include the amplitude of low-frequency fluctuation amplitude (ALFF) and seed-based functional connectivity.

After preprocessing the data, the ALFF will be calculated to compare the whole-brain spontaneous activity pattern before and after intervention in each group, as well as between patients with FC and HS following 10-week Tai Chi practices. The different regions obtained at ALFF analysis will be selected as the region of interest (ROI), also called seed, to perform the seed-based functional connectivity analysis and to explore the functional synchronization of ROI and other regions. The thresholds of $p<0.05$ with a false discovery rate correlation will be applied to all analyses. 
2.10.3. Correlation Analysis. To investigate the associations between nonspecific effects and central responses under different physical conditions, we performed the correlation analysis between the clinical data, including motor function, respiratory function, psychological state, and cerebral function in patients with FC and HS. In order to further explain the regulation mechanism of Tai Chi on the braingut interaction disorder, the correlation analysis among gastrointestinal symptoms, heart rate variability, and brain function activities in FC patients will also be carried out.

2.10.4. Safety Assessments. Adverse events might happen during Tai Chi practice, including strain, sprain, nausea, and dizziness. If any these adverse events occur, the instructor will take appropriate treatment according to clinician's advice and record the processing detail in the CRFs. The safety assessments will be monitored by the Ethics Committee.

The schedule of the study, including enrollment, interventions, assessments, and visits for participants, is shown in Table 1.

\section{Discussion}

As a popular physical-mind exercise, Tai Chi is widely used in the prevention, treatment, and rehabilitation of various diseases. On the basis of the characteristics and related studies on Tai Chi, we put forward the original hypothesis that Tai Chi practice can produce relative specific effects and nonspecific effects on the human body.

The main purposes of this study include two aspects. First, this study tries to explore the physiological and psychological existence of the specific and nonspecific effects of Tai Chi by comparing the differences in the motor function, respiratory function, gastrointestinal symptoms, psychological manifestations, and HRV between FC patients and HS after Tai Chi practice. Second, this study tries to explore the potential central mechanism of the specific and nonspecific effects of Tai Chi practice by analyzing the potential cerebral activity changes induced by Tai Chi and their correlation with clinical variables. The study will provide a new approach for investigating the mind-body exercise, and the results might deepen our knowledge about Tai Chi.

3.1. Specific and Nonspecific Effects of Tai Chi. Tai Chi is a complex sport that requires the coordination of spirit, breathing, postures, and movements. It can produce a significant impact on almost all human systems such as motor system, cardiovascular system, respiratory system, and digestive system. The impact is far beyond the physiological and psychological changes brought by a simple physical exercise. Thus, Tai Chi is not only widely used in public healthcare but also involved in disease treatment and rehabilitation.

On the basis of the characteristics of Tai Chi and related studies, we put forward that there are two types of effects of Tai Chi. One is the specific effect, which mainly indicates the therapeutic effects of Tai Chi practice on the pathological status and has obvious disease orientation. For instance, Tai Chi practice could produce a specificregulating effect on the abnormal gastrointestinal (GI) function of patients with GI disorders, specifically improve the cognitive function of patients with Alzheimer's disease [26], and significantly relieve the depression symptoms of patients with depression [27]. A systematic review and meta-analysis demonstrated that Tai Chi and other mind-body interventions were effective in alleviating GI symptoms and improving several aspects of the disease-related QOL including interference with activity, body image, health worry, food avoidance, and social reaction [10].

The second aspect is the nonspecific effects, which mainly refers to the benefits for the motor system, respiratory system, and others. No matter Tai Chi practice or other forms of physical exercises can produce it. The nonspecific effects might be a promoting effect on the motor system and respiratory system for not only the patients but also the HS. For example, studies demonstrated that Tai Chi exercises could increase the muscle strength in the lower extremities, improve balance control, and reduce the risk of falls for both healthy elderly people $[28,29]$ and Parkinson's and stroke patients [30, 31]. However, it should be emphasized that the specific effects of Tai Chi are relative and conditional. The nonspecific effects are the more widely used and fundamental effect of Tai Chi.

3.2. Potential Central Responses of Tai Chi. As a typical physical and mental exercise, Tai Chi not only emphasizes physical movement but also requires the state of unity of body and mind. The positive effects of meditation and aerobic exercise on cognitive function, including memory and decision-making abilities, have been widely accepted $[32,33]$. Recent studies further identified the influence of Tai Chi practice on the cerebral structure and function of practitioners [34]. For example, MRI studies showed that Tai Chi training evoked significant changes in the brain white matter network [35], brain volume [36], and spontaneous brain functional activities [37] in older people. These studies indicated that Tai Chi practice could influence not only the cerebral function but also the brain structure. It might be the central mechanism of the specific and nonspecific effects of Tai Chi.

In this study, FC patients will be enrolled to investigate the specific effects of Tai Chi-modulating GI function. FC, as a typical FGID, the dysfunction of the gut-brain axis plays an essential role in its pathogenesis [38]. Recent neuroimaging studies identified the abnormality in the cerebral function and structure in FC patients compared with the healthy controls. For example, an fMRI study demonstrated that FC patients showed significant differences in baseline brain activities in several brain regions implicated in emotional process modulation, somatic and sensory processing, and motor control regions [39]. Considering the existence of cerebral structural and functional abnormalities in FC patients, it is 
TABle 1: Study period.

\begin{tabular}{|c|c|c|c|c|c|c|}
\hline \multirow[b]{3}{*}{ Timepoint ${ }^{* *}$} & \multicolumn{6}{|c|}{ Study period } \\
\hline & \multirow{2}{*}{$\begin{array}{l}\text { Enrollment } \\
\text {-14 days }\end{array}$} & \multirow{2}{*}{$\begin{array}{l}\text { Allocation } \\
-7 \text { days }\end{array}$} & \multicolumn{4}{|c|}{ Postallocation } \\
\hline & & & $\begin{array}{c}0 \text { day } \\
\text { (baseline) }\end{array}$ & $\begin{array}{c}35 \text { days } \\
\text { (middle of intervention) }\end{array}$ & $\begin{array}{c}70 \text { days } \\
\text { (after intervention) }\end{array}$ & Etc. \\
\hline \multicolumn{7}{|l|}{ Enrollment } \\
\hline Eligibility screen & $x$ & & & & & \\
\hline Informed consent & $x$ & & & & & \\
\hline Demographics & $x$ & & & & & \\
\hline Diagnosis & $x$ & & & & & \\
\hline Past medical history & $x$ & & & & & \\
\hline Physical examination & & $x$ & & & & \\
\hline Allocation & & $x$ & & & & \\
\hline \multicolumn{7}{|l|}{ Interventions } \\
\hline Group A (FC patients) & & & $x$ & $x$ & $x$ & \\
\hline Group B (HS) & & & $x$ & $x$ & $x$ & \\
\hline \multicolumn{7}{|l|}{ Assessments } \\
\hline BMI & & $x$ & & & $x$ & \\
\hline $\mathrm{HRV}$ & & $x$ & & & $x$ & \\
\hline Lower-extremity muscle & & & $x$ & $x$ & $x$ & \\
\hline Functional balance & & & $x$ & $x$ & $x$ & \\
\hline Vital capacity & & & $x$ & $x$ & $x$ & \\
\hline Exercise self-rating scale & & & $x$ & $x$ & $x$ & \\
\hline CCS & & & $x$ & $x$ & $x$ & \\
\hline PAC-QOL & & & $x$ & $x$ & $x$ & \\
\hline PAC-SYM & & & $x$ & $x$ & $x$ & \\
\hline Stool diary & & & $x$ & $x$ & $x$ & \\
\hline SDS, SAS & & & $x$ & $x$ & $x$ & \\
\hline EPQ & & & $x$ & $x$ & $x$ & \\
\hline SF-36 & & & $x$ & $x$ & $x$ & \\
\hline MRI & & & $x$ & & $x$ & \\
\hline \multicolumn{7}{|l|}{ Safety observation } \\
\hline Blood routine test & & & $x$ & & & \\
\hline Urine routine test & & & $x$ & & & \\
\hline Stool routine test & & & $x$ & & & \\
\hline Adverse events & & & & & $x$ & \\
\hline \multicolumn{7}{|c|}{$\begin{array}{l}\text { This is a neuroimaging trial that includes a 2-week baseline period and a 10-week treatment period. In the baseline period, recruited patients will be screened } \\
\text { according to the inclusion criteria and exclusion criteria, and then, eligible FC patients and HS will sign an informed consent form and receive a physical } \\
\text { examination. After allocation, the FC patients and HS will be recruited and will receive } 10 \text { weeks of Tai Chi exercise. Schedule of enrollment, interventions, } \\
\text { and assessments: at the baseline, the median of intervention ( } 5 \text { weeks of exercise), and the end of the intervention (10 weeks of exercise). Among them, lower- } \\
\text { extremity muscle strength was measured using a hand-held isometric dynamometer (Micro FET3; Hoggan Health Industries). Functional balance tests } \\
\text { include BBS, TUG test, and functional reach test. During the vital capacity test, FEV1, FVC, and FEV1/FVC ratio were measured using a MicroMedical Super } \\
\text { Spiro spirometer in resting status. PEFR was assayed using a peak flow meter. The stool diary, CCS, and PAC-SYM will be used to evaluate the clinical efficacy } \\
\text { of different interventions; the PAC-QOL will be used to assess the health-related QOL; the SDS, SAS, EPQ, and Mini-Mental State Examinations will be used } \\
\text { to consider the effect of psychological factors on the patients' symptoms. All participants will be assessed during the } 24 \mathrm{~h} \text { HRV to evaluate the autonomic } \\
\text { nervous function at the baseline and the end of the 10-week intervention. fMRI scans will be performed to detect the cerebral functional changes in } 15 \text { patients } \\
\text { in each group both at the baseline and the end of the intervention. }\end{array}$} \\
\hline
\end{tabular}

feasible to explore the mechanism of Tai Chi's specific effects on GI function of FC patients.

In this study, fMRI will be selected as the neuroimaging technique to investigate the cerebral activity changes elicited by Tai Chi practice. fMRI is the most commonly used brain imaging technique and has been widely used in the studies on the central mechanism of Tai Chi intervention $[35,40,41]$. For example, using fMRI, people found that influencing the resting-state functional connectivity alteration between the cognitive control network and rostral anterior cingulate cortex and medial prefrontal cortex might be the central mechanism of Tai Chi intervention for fibromyalgia [41]. These studies indicated that it was reliable to use fMRI to explore the central mechanism of Tai Chi intervention for FC.

Furthermore, to ensure the reliability and repeatability of the results, we will establish a strict quality control program, which includes the participant selection, the SOP of Tai Chi practice, the SOP of neuroimaging scan, and the outcome measures. For example, for the selection of participants, the freshmen who never practice Tai Chi and have no physical exercise frequently will be taken into consideration. In the implementation of Tai Chi intervention, the SOP of 24-style Tai Chi is established, which covers the requirement on the movements, breathing, and ideas of the participant. 


\section{Conclusions}

In conclusion, Tai Chi is a safe and effective assistant therapy for many diseases. However, whether the therapeutic effects of Tai Chi are disease-oriented remains uncertain. This trial is the first to investigate the existence of the specific and nonspecific effects of Tai Chi and to explore their potential central responses. It will provide a new perspective for understanding Tai Chi and a new approach for studying mind-body exercise.

\section{Abbreviations}

HS: $\quad$ Healthy subject

FC: $\quad$ Functional constipation

FGID: $\quad$ Functional gastrointestinal disease

QOL: Quality of life

GI: $\quad$ Gastrointestinal

fMRI: $\quad$ Functional magnetic resonance imaging

CDUTCM: Chengdu University of Traditional Chinese Medicine

CSBMs: Complete spontaneous bowel movements

CCS: Cleveland Constipation Score

RPE: $\quad$ Rating of perceived exertion

PAC-SYM: Patient Assessment of Constipation Symptom

PAC-QOL: Patient Assessment of Constipation Quality of Life Questionnaire

RPE: $\quad$ Rating of perceived exertion

SF-36: The MOS 36-item short-form health survey

BBS: $\quad$ Berg Balance Scale

TUG: $\quad$ Timed up-and-go

FEV1: $\quad$ Forced expiratory volume in one second

FVC: $\quad$ Forced vital capacity

SDS: $\quad$ Self-Rating Depression Scale

SAS: $\quad$ Self-Rating Anxiety Scale

EPQ: $\quad$ Eysenck Personality Questionnaires

HRV: $\quad$ Heart rate variability

3D-T1WI: 3-dimensional T1-weighted imaging

BOLD- Blood oxygenation level-dependent fMRI

fMRI:

DTI: Diffusion tensor imaging

CRF: $\quad$ Case report form

ITT: Intention to treat

PP: $\quad$ Per protocol

ReHo: Regional homogeneity

ALFF: Amplitude of low-frequency fluctuation amplitude.

\section{Data Availability}

This is a protocol for a clinical trial, and no original data are included.

\section{Ethical Approval}

The procedures have been approved by the Sichuan Regional Ethics Review Committee on Traditional Chinese Medicine (no. 2018KL-047) and conformed to the Declaration of Helsinki. In addition, all researchers were trained and signed a pledge to protect the confidentiality of study participants.

\section{Consent}

All enrolled subjects signed an informed consent form.

\section{Disclosure}

Tianyu Liu, Yuke Teng, and Sha Yang are the co-first authors.

\section{Conflicts of Interest}

The authors declare that they have no conflicts of interest.

\section{Authors' Contributions}

Tianyu Liu, Yuke Teng, and Sha Yang contributed equally to this article. FZ and JW were responsible for this study. TL, YT, SY, FZ, JW, ZH, and SY conceived and designed the study. TL, YT, and SY participated in drafting the trial protocol and preparing the manuscript. YG, TY, JC, and RY participated in data collection and were in charge of recruitment and treatment of patients. All authors read and approved the final manuscript.

\section{Acknowledgments}

This study was supported by the Humanities and Social Sciences Youth Project of the Ministry of Education of China (19YJC890027). The trial was financially supported by the Ten-Thousand Talents Program of China (W02020595) and the China Postdoctoral Science Foundation (2020M673562XB).

\section{References}

[1] C. Lan, J.-S. Lai, S.-Y. Chen, and M.-K. Wong, "Tai Chi Chuan to improve muscular strength and endurance in elderly individuals: a pilot study," Archives of Physical Medicine and Rehabilitation, vol. 81, no. 5, pp. 604-607, 2000.

[2] A. M. K. Wong and C. Lan, "Tai Chi and balance control," Medicine and Sport Science, vol. 52, pp. 115-123, 2008.

[3] L. Sun, L. P. Zhuang, X. Z. Li, J. Zheng, and W. F. Wu, "Tai Chi can prevent cardiovascular disease and improve cardiopulmonary function of adults with obesity aged 50 years and older: a long-term follow-up study," Medicine, vol. 98, no. 42, Article ID e17509, 2019.

[4] C. E. Rogers, L. K. Larkey, and C. Keller, "A review of clinical trials of Tai Chi and Qigong in older adults," Western Journal of Nursing Research, vol. 31, no. 2, pp. 245-279, 2009.

[5] J. H. Yan, W. J. Gu, J. Sun, W. X. Zhang, B. W. Li, and L. Pan, "Efficacy of Tai Chi on pain, stiffness and function in patients with osteoarthritis: a meta-analysis," PLoS One, vol. 8, no. 4, Article ID e61672, 2013.

[6] C. Wang, J. P. Collet, and J. Lau, "The effect of Tai Chi on health outcomes in patients with chronic conditions: a systematic review," Archives of Internal Medicine, vol. 164, no. 5, pp. 493-501, 2004.

[7] M. Chao, C. Wang, X. Dong, and M. Ding, "The effects of tai chi on type 2 diabetes mellitus: a meta-analysis," Journal of Diabetes Research, vol. 2018, Article ID 7350567, 9 pages, 2018. 
[8] E. Salmoirago-Blotcher, P. M. Wayne, S. Dunsiger et al., "Tai Chi is a promising exercise option for patients with coronary heart disease declining cardiac rehabilitation," Journal of the American Heart Association, vol. 6, no. 10, 2017.

[9] L. Wang, K. Wu, X. Chen, and Q. Liu, "The effects of tai chi on lung function, exercise capacity and health related quality of life for patients with chronic obstructive pulmonary disease: a pilot study," Heart, Lung and Circulation, vol. 28, no. 8, pp. 1206-1212, 2019.

[10] W. Wang, F. Wang, F. Fan, A. C. Sedas, and J. Wang, "Mindbody interventions for irritable bowel syndrome patients in the Chinese population: a systematic review and meta-analysis," International Journal of Behavioral Medicine, vol. 24, no. 2, pp. 191-204, 2017.

[11] Y. Zhou, L. Liang, L. I. Hong, and L. Jing, "About the convalescent influence of 24-Pattern Shadowboxing to the patients with chronic functional constipation," Liaoning Sport Science and Technology, vol. 2, pp. 52-54, 2015.

[12] P. Katelaris, V. Naganathan, K. Liu, G. Krassas, and J. Gullotta, "Comparison of the effectiveness of polyethylene glycol with and without electrolytes in constipation: a systematic review and network meta-analysis," BMC Gastroenterology, vol. 16, no. 1, p. 42, 2016.

[13] S. C. Chow, J. Shao, and H. Wang, Sample Size Calculations in Clinical Research, Wiley, Hoboken, NJ, USA, 2003.

[14] D. A. Drossman and W. L. Hasler, "Rome IV-functional GI disorders: disorders of gut-brain interaction," Gastroenterology, vol. 150, no. 6, pp. 1257-1261, 2016.

[15] S. Yang, J. Chen, Y. Guo et al., "Comparison of Taiji and aerobic exercise for functional constipation: study protocol for a randomised controlled neuroimaging trial," BMJ Open, vol. 9, no. 8, Article ID e031089, 2019.

[16] K. Berg, S. Wood-Dauphinee, and J. I. Williams, "The balance scale: reliability assessment with elderly residents and patients with an acute stroke," Scandinavian Journal of Rehabilitation Medicine, vol. 27, no. 1, pp. 27-36, 1995.

[17] P. W. Duncan, D. K. Weiner, J. Chandler, and S. Studenski, "Functional reach: a new clinical measure of balance," Journal of Gerontology, vol. 45, no. 6, pp. M192-M197, 1990.

[18] W. W. K. Zung, "A self-rating depression scale," Archives of General Psychiatry, vol. 12, no. 1, pp. 63-70, 1965.

[19] W. W. K. Zung, "A rating instrument for anxiety disorders," Psychosomatics, vol. 12, no. 6, pp. 371-379, 1971.

[20] D. Schuldberg, "Eysenck personality questionnaire scales and paper-and-pencil tests related to creativity," Psychological Reports, vol. 97, no. 1, pp. 180-182, 2005.

[21] F. Agachan, T. Chen, J. Pfeifer, P. Reissman, and S. D. Wexner, "A constipation scoring system to simplify evaluation and management of constipated patients," Diseases of the Colon \& Rectum, vol. 39, no. 6, pp. 681-685, 1996.

[22] L. Frank, L. Kleinman, C. Farup, L. Taylor, and P. Miner Jr., "Psychometric validation of a constipation symptom assessment questionnaire," Scandinavian Journal of Gastroenterology, vol. 34, no. 9, pp. 870-877, 1999.

[23] P. Marquis, C. De La Loge, D. Dubois, A. McDermott, and O. Chassany, "Development and validation of the patient Assessment of constipation quality of life questionnaire," Scandinavian Journal of Gastroenterology, vol. 40, no. 5, pp. 540-551, 2005.

[24] S. Morishita, A. Tsubaki, T. Takabayashi, and J. B. Fu, "Relationship between the rating of perceived exertion scale and the load intensity of resistance training," Strength and Conditioning Journal, vol. 40, no. 2, pp. 94-109, 2018.
[25] A. Kelly, J. Rush, E. Shafonsky et al., "Detecting short-term change and variation in health-related quality of life: withinand between-person factor structure of the SF-36 health survey," Health and Quality of Life Outcomes, vol. 13, no. 1, p. 199, 2015.

[26] P. J. Klein, “Tai Chi Chuan in the management of Parkinson's disease and Alzheimer's disease," Medicine and Sport Science, vol. 52, pp. 173-181, 2008.

[27] J. Kong, G. Wilson, J. Park, K. Pereira, C. Walpole, and A. Yeung, "Treating depression with Tai Chi: state of the art and future perspectives," Front Psychiatry, vol. 10, p. 237, 2019.

[28] W. W. N. Tsang and C. W. Y. Hui-Chan, "Effect of 4- and 8wk intensive Tai Chi training on balance control in the elderly," Medicine \& Science in Sports \& Exercise, vol. 36, no. 4, pp. 648-657, 2004.

[29] Z. G. Huang, Y. H. Feng, Y. H. Li, and C. S. Lv, "Systematic review and meta-analysis: Tai Chi for preventing falls in older adults," BMJ Open, vol. 7, no. 2, Article ID e013661, 2017.

[30] F. Li, P. Harmer, K. Fitzgerald et al., "Tai Chi and postural stability in patients with Parkinson's disease," New England Journal of Medicine, vol. 366, no. 6, pp. 511-519, 2012.

[31] I. Hwang, R. Song, S. Ahn, M.-A. Lee, P. M. Wayne, and M. K. Sohn, "Exploring the adaptability of Tai Chi to stroke rehabilitation," Rehabilitation Nursing, vol. 44, no. 4, pp. 221-229, 2019.

[32] S. Deepeshwar, S. A. Vinchurkar, N. K. Visweswaraiah, and H. R. Nagendra, "Hemodynamic responses on prefrontal cortex related to meditation and attentional task," Frontiers in Systems Neuroscience, vol. 8, p. 252, 2014.

[33] T. Moriarty, K. Bourbeau, B. Bellovary, and M. N. Zuhl, "Exercise intensity influences prefrontal cortex oxygenation during cognitive testing," Behavioral Sciences, vol. 9, no. 8, p. 83, 2019.

[34] R. Silveira, R. C. R. Prado, C. Brietzke et al., "Prefrontal cortex asymmetry and psychological responses to exercise: a systematic review," Physiology \& Behavior, vol. 208, p. 112580, 2019.

[35] C. Yue, L. Zou, J. Mei et al., "Tai Chi training evokes significant changes in brain white matter network in older women," Healthcare, vol. 8, no. 1, p. 57, 2020.

[36] M. Adcock, M. Fankhauser, J. Post et al., "Effects of an inhome multicomponent exergame training on physical functions, cognition, and brain volume of older adults: a randomized controlled trial," Frontiers in Medicine, vol. 6, p. 321, 2019.

[37] Z. Zheng, X. Zhu, S. Yin et al., "Combined cognitive-psychological-physical intervention induces reorganization of intrinsic functional brain architecture in older adults," Neural Plasticity, vol. 2015, Article ID 713104, 11 pages, 2015.

[38] M. Equit, J. Niemczyk, A. Kluth, C. Thomas, M. Rubly, and A. von Gontard, "Central nervous system processing of emotions in children with fecal incontinence and constipation," Zeitschrift für Kinder- und Jugendpsychiatrie und Psychotherapie, vol. 47, no. 1, pp. 67-71, 2019.

[39] Q. Zhu, W. Cai, J. Zheng et al., "Distinct resting-state brain activity in patients with functional constipation," Neuroscience Letters, vol. 632, pp. 141-146, 2016.

[40] J. Kong, E. Wolcott, Z. Wang et al., "Altered resting state functional connectivity of the cognitive control network in fibromyalgia and the modulation effect of mind-body intervention," Brain Imaging and Behavior, vol. 13, no. 2, pp. 482-492, 2019. 
[41] R. Jalilianhasanpour, E. Beheshtian, G. Sherbaf, S. Sahraian, and H. I. Sair, "Functional connectivity in neurodegenerative disorders: Alzheimer's disease and frontotemporal dementia," Topics in Magnetic Resonance Imaging, vol. 28, no. 6, pp. 317-324, 2019. 